\title{
Microsymposium
}

MS124.004

\section{Electron pairing over domains}

Miroslav Kohout ${ }^{1}$

${ }^{1}$ Max Planck Institute For Chemical Physics Of Solids, Dresden, Germany

E-mail: kohout@cpfs.mpg.de

The chemical bond is usually attributed to the pairing of electrons. The determination of the localization and delocalization of electrons, based in integrals describing the electron pairing within and between specific domains, yields important insight into the nature of chemical bonds [1,2]. Thus, the utilization of the corresponding indicators and its impact on the viewpoint on the chemical bond strongly depends on the chosen space partitioning. Additionally, the symmetry of the system as well as the inclusion of electron correlation can have significant influence on the meaning of the results.

[1] A. Martin Pendas, M. Kohout, M.A. Blanco, E. Francisco Beyond standard charge density topological analyses; In: Modern Charge Density Analysis, C. Gatti and P. Macchi (Eds), Springer, I ISBN 978-90-481-3835-7, Springer, Dordrecht Heidelberg London New York, 2012

[2] M. Kohout Electron pairs in position space, Struct Bond 170, 119-168 (2016)

Keywords: delocalization, pair density 